\title{
Research on Service Life of Flame Resistant Materials in the Exhibition Hall by the Use of Mechanical Method of the Meso Damage Mechanics
}

\author{
Junzhu Zhang \\ Shunde Polytechnic, Foshan, Guangdong, China
}

\begin{abstract}
The population in the exhibition hall is relatively dense, and fire incidents often occur, so the existence of flame resistant materials is very important. The flame resistant materials are used to analyze the museums, art galleries, science and technology museums and other exhibition halls. Taking two kinds of flame resistant materials, namely, $\mathrm{Mg}(\mathrm{OH})_{2}$ and $\mathrm{Al}(\mathrm{OH})_{3}$ as an example, this paper establishes a model of meso damage mechanics by the use of mechanical method of the meso damage mechanics, and researches the macro mechanical properties of the thermal insulation materials and decorative materials so as to predict its service life. This research finds that the use of two kinds of flame resistant materials, namely, $\mathrm{Mg}(\mathrm{OH})_{2}$ and $\mathrm{Al}(\mathrm{OH})_{3}$ can improve the elasticity modulus of the thermal insulation materials and decorative materials used in the construction, so that its macro mechanical properties can have a significant improvement, and its service life can also have a significant improvement after adding flame retardant materials.
\end{abstract}

Keywords: exhibition hall; flame retardant materials; meso damage mechanics

\section{PREFACE}

At the current stage, the flame retardant materials have been widely used in metallurgy, textile, architecture, fire prevention and other major industries, and the flame retardant materials also have been used in the national defense.

Lai Xuejun has researched the flame resistant polypropylene, and introduced the preparation process of efficient water-resistant flame retardant in detail, and indicated that the preparation process of the flame retardant materials must strictly abide by the flame retardant mechanism of the flame retardant. Yang $\mathrm{Li}$ has also carried out an in-depth discussion on this issue. The flame retardant mechanism is related to the types of the flame retardant. The flame retardant mechanism of the solid phase, gas phase and liquid phase is different, which must be fully considered during the preparation.

The meso mechanics is not only a kind of common method to research the link between the micro structure and macro properties, but also an effective method. Huang Jianke analyzes the fracture criterion in metal forming, and analyzes the microstructure in metal forming process by the use of meso damage mechanics, and eventually finds its ductile change rules; Liu Xianlan also proposes that how to effectively observe and measure metal plasticity is very important in the process of metal plastic processing, while the application of the meso damage mechanics provides it with an effective way.

There are many problems that are required to being considered in the process of building a large-scale exhibition hall. The use and selection of materials is crucial. Guo Donghai points out that the fireproof ma- terials must be used in the architectural design of the exhibition hall so as to avoid fire. Therefore, the application of flame retardant materials is very important.

This paper researches an important role of the flame retardant materials in building an exhibition hall by the use of the mechanical method of the meso damage mechanics, and analyzes the macro mechanical properties of the thermal insulation materials and decorative materials used for the architecture, and eventually predicts its service life.

\section{PRINCIPLE ANALYSIS}

\subsection{Flame retardant materials}

As the literal meaning of this given name, the flame retardant material is a kind of material that can effectively prevent or delay combustion, which is not prone to burn due to its low ignition point. As a kind of protective material, the flame retardant material has a variety of types, including solid and liquid materials. Generally, the solid flame retardant materials are a variety of glass, cement, steel and so on. The liquid flame retardant material is actually flame-retardant, which is sprayed on various fire-fighting materials or the wall surface, and its main mechanism is to prevent the wall or materials from burning in case of catching fire so as to reduce the scope of combustion.

The flame retardant materials can be not only divided into organic and inorganic materials, but also halogen and non-halogen materials. High flame retardant materials have many types. High organic flame retardant materials are generally represented by the nitrogen-containing flame, red phosphorus and com- 


\section{MATEC Web of Conferences}

pounds, and bromine-containing flame; high inorganic flame retardant materials are mainly antimonous oxide, $\mathrm{Mg}(\mathrm{OH})_{2}$ and $\mathrm{Al}(\mathrm{OH})_{3}$, silicon-containing flame and so on.

The flame retardant ways of the flame retardant materials are different, which generally include the following ways:

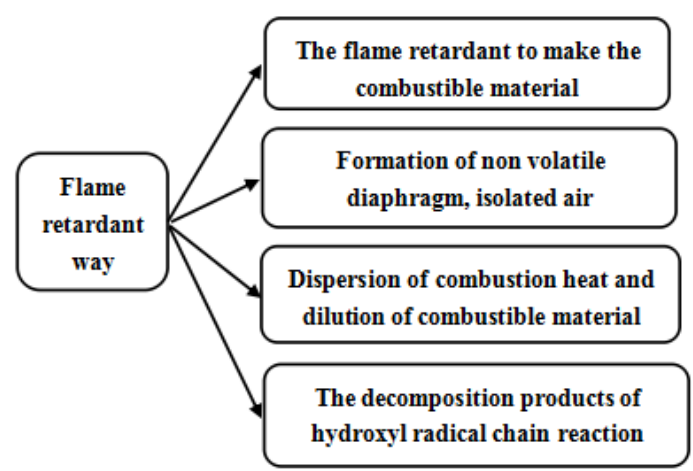

Figure 1. Flame retardant way

(1) It can be used to promote the carbonization of combustible material and prevent burning, and ultimately achieve the purpose of flame retarding. The phosphorus-based flame retardant often plays a role in the solid phase by the use of the carbonation role of the combustible material;

(2) It can form non-volatile diaphragm by using the function of the flame retardant and isolate air then achieve the effect of flame retarding. In the process of using the halide, antimony oxide, borate, phosphorus-based materials and other liquid-phase flame retardant materials, the effect of flame retarding is usually very obvious;

(3) The products decomposed by the flame retardant can obstruct the chain reaction between $\mathrm{H}$ and $\mathrm{O}$ so as to prevent combustion. In the process of using halide and antimony oxide flame-retardant materials, it will play a role in the gas phase;

(4) To disperse combustion heat and dilute combustible material. Generally, in the process of using $\mathrm{Mg}(\mathrm{OH})_{2}$ and $\mathrm{Al}(\mathrm{OH})_{3}$ and other flame retardant materials, it will absorb a lot of heat due to decomposition and other chemical reactions. Its chemical products are non-combustible materials, which will prevent the combustibles burning under these two effects.

\subsection{Application of flame retardant materials into the buildings of exhibition halls}

For large-scale buildings, the fire prevention measures must be done. In recent years, there are many fire incidents in the large-scale buildings. "Fire" always threatens our personal safety. As the most densely-populated exhibition hall, it receives thousands of tourists every day, so the most important task for safety of the exhibition hall is the fire prevention.
In the process of building the exhibition halls, museums, art galleries, science and technology museums, fire prevention measures must be in place. High flame retardant materials must be adopted, and the flame retardant must be sprayed on the fireproof wall. Only when all-round work is in place can the fire be prevented before it happens.

First, in the process of building museums, art galleries, the science and technology museums or other buildings, the energy saving and insulation polymer materials can not only benefit them, but also induce fire incidents. Among them, the energy saving and insulation polyphenol vinegar materials, electric wire and cable and other polyolefin materials used inside the buildings of museums, art galleries or the science and technology museums are fire incentives. Therefore, the flame retardant treatment in these parts is very important.

Second, in the process of building museums, art galleries, the science and technology museums or other buildings, some decorative materials may be used. There exist serious safety problems in many interior decorations which are made of combustible materials, such as wood furniture, plastic products and glass products used by light fixtures, as well as curtains and other textile products and so on. In general, it is necessary to adopt the flame retardant technology and flame retardant materials for these textile products.

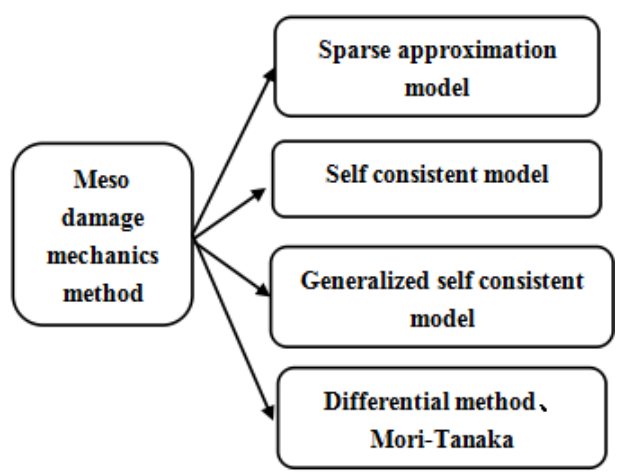

Figure 2. Mechanical method of the meso damage mechanics

Combined with the types and characteristics of flame retardant materials, taking into account practical problems in the process of building above exhibition halls, it is very necessary to select appropriate flame retardant materials or flame retardants. $\mathrm{Mg}(\mathrm{OH})_{2}$ and $\mathrm{Al}(\mathrm{OH})_{3}$ as inorganic flame retardants when used for the preparation of flame retardant materials, their flame retardant mechanism relies on their chemical reaction to absorb heat, and relies on the non-combustible products in the chemical reaction to decompose combustibles so as to disperse heat of combustion, and achieve the effect of flame retarding. This way can not only retard flame, but also inhibit the formation of smoke. In addition, $\operatorname{Mg}(\mathrm{OH})_{2}$ and $\mathrm{Al}(\mathrm{OH})_{3}$ have a good stability without corrosivity, 


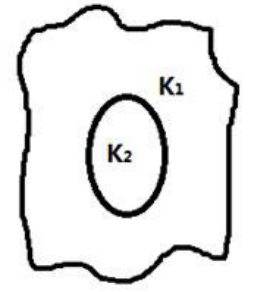

(a)Sparse approximation model

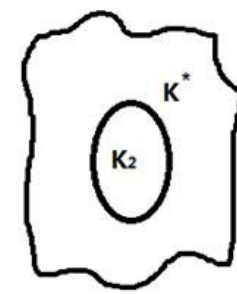

(b)Self consistent model

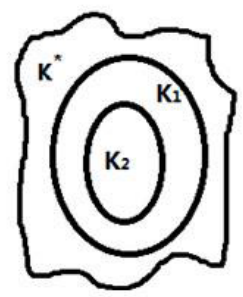

(c) Generalized self consistent model

Figure 3. Schematic diagram of common micro mechanical model

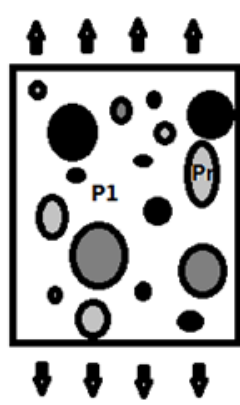

Inhomogeneous medium

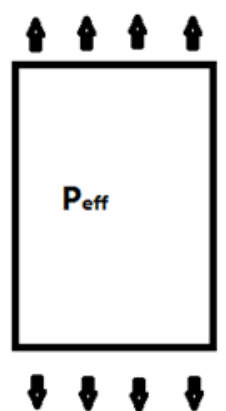

Equivalent homogeneous medium

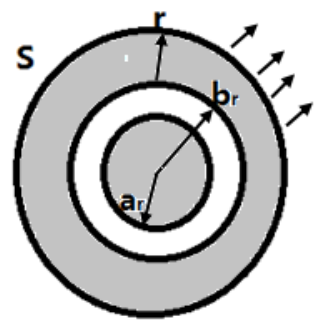

Figure 4. Generalized self-consistent model

which does not release toxic gases at high temperatures with a wide source and acceptable price, so it is a more widely-used type among the flame retardant materials.

On this basis, this paper researches the damage properties of two kinds of flame retardants, namely, $\mathrm{Mg}(\mathrm{OH})_{2}$ and $\mathrm{Al}(\mathrm{OH})_{3}$ when they are applied to the insulation materials and decorative materials of museums, art galleries, the science and technology museums or other buildings, thereby predicting the service life of museums, art galleries, the science and technology museums or other exhibition halls.

\section{EXPERIMENT SIMULATION}

\subsection{Mechanical method of the meso damage me- chanics}

What is meso damage mechanics? It is to research the macro damage behavior and evolution process of the materials from the meso structure. Among them, the meso structure of the materials includes particles and opening or holes of the materials under the microscopic form, while the damage of the macro mechanical properties is reflected in its surface cracks or breakage and so on.

The common mechanical methods of the meso damage mechanics are as Figure 2.

The commonly-used mechanical methods of the meso damage mechanics are the sparse approximation model, self-consistent model and generalized self-consistent model. The schematic diagrams of these models are as Figure 3.

In the three methods in Figure 3, the generalized self-consistent model overcomes the shortcomings of the other two models, which is more perfect. With the matrix of an infinite equivalent medium, it embeds the mixed and matrical composite ball in it so as to give a reasonable prediction on a variety of extreme phenomena. Therefore, this paper carries out a research mainly by using this method. The following is a detailed working principle of this method.

Taking two kinds of flame resistant materials, namely, $\mathrm{Mg}(\mathrm{OH})_{2}$ and $\mathrm{Al}(\mathrm{OH})_{3}$ as an example, this paper researches the damage behavior and macro mechanical properties of $\mathrm{Mg}(\mathrm{OH})_{2}$ and $\mathrm{Al}(\mathrm{OH})_{3}$ through the generalized self-consistent model when they are applied to the insulation materials and decorative materials in museums, art galleries, the science and technology museums or other buildings, thereby predicting the service life of museums, art galleries, the science and technology museums or other exhibition halls.

\subsection{Experiment simulation}

The model method of meso damage mechanics can be used to research the insulation materials and decorative materials, and analyze its macro mechanical 


\section{MATEC Web of Conferences}

properties, thereby predicting the service life of museums, art galleries, the science and technology museums or other exhibition halls. The prediction process is as follows:

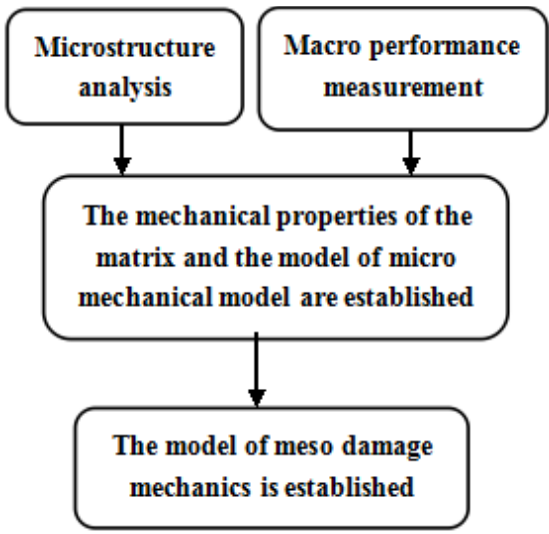

Figure 5. Establishment of meso damage mechanics model

$\mathrm{Mg}(\mathrm{OH})_{2}$ and $\mathrm{Al}(\mathrm{OH})_{3}$ are often considered as the matrix phase of the insulation materials and decorative materials to research. To research the macro mechanical properties of the matrix phase, there is a first need to know the typical microstructure of insulation materials and decorative materials, which is shown as follows:
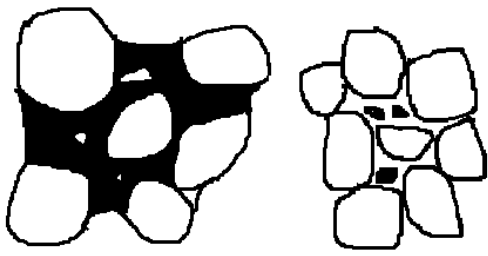

Figure 6. Typical microstructure of materials

Here, we choose two kinds of materials, namely, the thermal insulation material (A) and the decorative material (B) as the research objects. Among them, $\mathrm{Mg}(\mathrm{OH})_{2}$ is the matrix phase of the thermal insulation material $(\mathrm{A})$, while $\mathrm{Al}(\mathrm{OH})_{3}$ is the matrix phase of the decorative material (B).The phase composition and mechanical properties are shown in Table 1 and Table

Table 1. Composition of two kinds of materials

\begin{tabular}{|l|l|l|}
\hline & $\begin{array}{l}\text { Material A } \\
\mathrm{Mg}(\mathrm{OH})_{2}\end{array}$ & $\begin{array}{l}\text { Material B } \\
\mathrm{Al}(\mathrm{OH})_{3}\end{array}$ \\
\hline Chemical composition & $78.4 \%$ & $91.8 \%$ \\
Aggregate & $63 \%$ & $84 \%$ \\
\hline Continuous phase & $32 \%$ & $16 \%$ \\
\hline
\end{tabular}

The elastic properties of the matrix phases $\mathrm{Mg}(\mathrm{OH})_{2}$ and $\mathrm{Al}(\mathrm{OH})_{3}$ of two kinds of materials, namely, the insulation material (A) and the decorative material (B) can be predicted by the generalized self-consistent model. The results are shown in the Table 3.

Table 2. Normal-temperature pressure tests of two kinds of materials

\begin{tabular}{|l|l|l|}
\hline & Material A & Material B \\
& $\mathrm{Mg}(\mathrm{OH})_{2}$ & $\mathrm{Al}(\mathrm{OH})_{3}$ \\
\hline Elasticity modulus & 22.3 & 89 \\
$E(\mathrm{GPa})$ & & 0.4 \\
\hline Poisson's ratio v & 0.4 & -105 \\
\hline Strength $\sigma(\mathrm{MPa})$ & -35.7 & \\
\hline
\end{tabular}

Table 3. Prediction results of the matrix phase properties

\begin{tabular}{|l|l|l|}
\hline & $\begin{array}{l}\text { Material A } \\
\mathrm{Mg}(\mathrm{OH})_{2}\end{array}$ & $\begin{array}{l}\text { Material B } \\
\mathrm{Al}(\mathrm{OH})_{3}\end{array}$ \\
\hline $\begin{array}{l}\text { Elasticity modulus } \\
E \text { (GPa) }\end{array}$ & 6 & 11.56 \\
\hline Poisson's ratio v & 0.23 & 0.35 \\
\hline
\end{tabular}

Hence, the stress strain curves of the two kinds of materials are as follows:

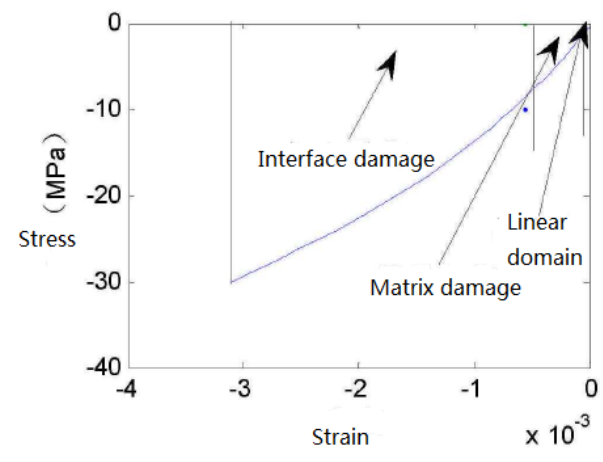

Figure 7. Stress strain curve

Thus Young's modulus strain curve of two kinds of materials (A and $\mathrm{B}$ ) can be obtained. To divide Young's modulus and strain curve into the matrix damage stage and interface damage stage, the following relation curve can be obtained:

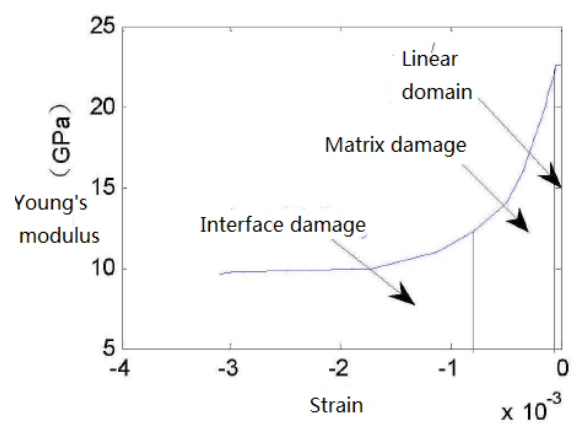

Figure 8. Young's modulus strain curve 
The above analysis shows that the damage behavior of the two kinds of materials (A and $\mathrm{B}$ ) depends on the properties of the matrix phases $\mathrm{Mg}(\mathrm{OH})_{2}$ and $\mathrm{Al}(\mathrm{OH})_{3}$, or the properties of the interface phase. Among them, the properties of the matrix phases $\mathrm{Mg}(\mathrm{OH})_{2}$ and $\mathrm{Al}(\mathrm{OH})_{3}$ have a great impact on the macro properties of the materials (A and B), which are reflected in the Young's modulus strain curve.

The above research shows that the application of flame retardant in the insulation materials and decorative materials, namely, the use of $\mathrm{Mg}(\mathrm{OH})_{2}$ and $\mathrm{Al}(\mathrm{OH})_{3}$ can improve the elasticity modulus, and significantly improve the macro mechanical properties, which can be seen from the Young's modulus stress strain curve.

In order to further predict the service life of museums, art galleries, the science and technology museums or other exhibition halls in the use of flame retardant materials, now, the modified exponential curve is introduced, and the further research is carried out on this basis.

The mathematical model of the modified exponential curve is:

$\hat{y}_{t}=K+a b^{t}$

When the value of $K$ can be determined, the least square method can be used to determine the parameters of the model; when the value of $K$ fails to be predetermined, the three-sum method can be used. $n$ observed values of the time series are divided into three parts, of which each part has $m$ period(s), that is, $n=3 m$.

Party 1: $y_{1}, y_{2}, y_{3}, \cdots, y_{m}$;

Party 2: $y_{m+1}, y_{m+2}, y_{m+3}, \cdots, y_{2 m}$;

Party 3: $y_{2 m+1}, y_{2 m+2}, y_{2 m+3}, \cdots, y_{3 m}$

Among them, the sum of tendency in each part is equal to the sum of the corresponding observed values, thereby providing the parameter estimation.

The steps of the three-sum method are as follows:

The sum of the observed values in each part is:

$S_{1}=\sum_{t=1}^{m} y_{t}, S_{2}=\sum_{t=m+1}^{2 m} y_{t}, S_{3}=\sum_{t=2 m+1}^{3 m} y_{t}$,

And

$\left\{\begin{array}{l}S_{1}=\sum_{t=1}^{m} \hat{y}_{t}=\sum_{t=1}^{m}\left(K+a b^{t}\right)=m K+a b\left(1+b+b^{2}+\cdots+b^{m-1}\right) \\ S_{2}=\sum_{t=m+1}^{2 m} \hat{y}_{t}=\sum_{t=m+1}^{2 m}\left(K+a b^{t}\right)=m K+a b^{m+1}\left(1+b+b^{2}+\cdots+b^{m-1}\right) \\ S_{3}=\sum_{t=2 m+1}^{3 m} \hat{y}_{t}=\sum_{t=2 m+1}^{3 m}\left(K+a b^{t}\right)=m K+a b^{2 m+1}\left(1+b+b^{2}+\cdots+b^{m-1}\right)\end{array}\right.$

Where: $\left(1+b+b^{2}+\cdots+b^{m-1}\right)(b-1)=b^{m}-1$

Thereby obtain:

$$
\left\{\begin{array}{l}
S_{1}=m K+a b \frac{b^{m-1}}{b-1} \\
S_{2}=m K+a b^{m+1} \frac{b^{m-1}}{b-1} \\
S_{3}=m K+a b^{2 m+1} \frac{b^{m-1}}{b-1}
\end{array}\right.
$$

Thus:

$$
\left\{\begin{array}{l}
b=\left(\frac{S_{3}-S_{2}}{S_{2}-S_{1}}\right)^{\frac{1}{m}} \\
a=\left(S_{2}-S_{1}\right) \frac{b-1}{b\left(b^{m}-1\right)^{2}} \\
K=\frac{1}{m}\left[S_{1}-\frac{a b\left(b^{m}-1\right)}{(b-1)}\right]
\end{array}\right.
$$

Thus the service life of museums, art galleries, the science and technology museums or other exhibition halls can be predicted in the use of flame retardant materials. The service life prediction curve is as follows:

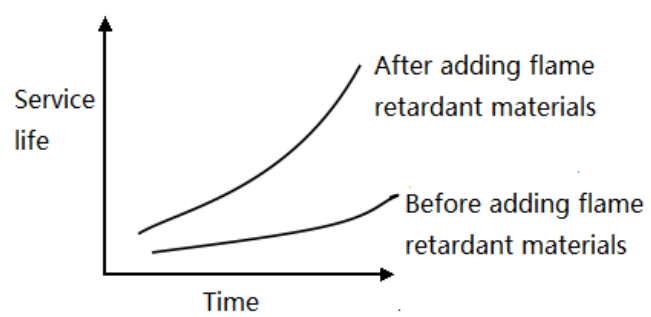

Figure 9. Service life prediction curve

Compared with not adding flame retardant materials before, the service life of museums, art galleries, the science and technology museums or other exhibition halls has a significant improvement with a faster and more significant improvement after adding flame retardant materials. It indicates that the use of flame retardant materials has a certain impact on the service life of museums, galleries, the science and technology museums or other exhibition halls.

\section{CONCLUSION}

Taking $\mathrm{Mg}(\mathrm{OH})_{2}$ and $\mathrm{Al}(\mathrm{OH})_{3}$ as an example, this paper establishes a model of meso damage mechanics by the using mechanical method of the meso damage mechanics through analysis of the flame retardant materials used in museums, art galleries, the science and technology museums or other exhibition halls, and researches the macro mechanical properties of the thermal insulation materials and decorative materials so as to predict their service life. 


\section{MATEC Web of Conferences}

(1) Through the establishment of the model of meso damage mechanics, the use of two kinds of flame resistant materials, namely, $\mathrm{Mg}(\mathrm{OH})_{2}$ and $\mathrm{Al}(\mathrm{OH})_{3}$ can improve the elasticity modulus of the thermal insulation materials and decorative materials used in the construction, so that its macro mechanical properties can have a significant improvement, which can be seen from the Young's modulus stress strain curve;

(2) The service life of museums, art galleries, the science and technology museums or other exhibition halls can be predicted in the use of flame retardant materials through the modified exponential curve. And it is found out that compared with not adding flame retardant materials before, the service life is significantly improved after adding them.

\section{REFERENCES}

[1] Lai Xuejun. 2012. Preparation of efficient water-resistan intumescent flame retardant and research of its flame resistant polypropylene. South China University of Technology.

[2] Yang Li. 2010. Discussion of flame resistant mechanism of the flame retardant. Tianjin Chemical.
[3] Ou Yuxiang. 2003. Development trend of flame retardant abroad and suggestion on development of flame retardant industry in China. Fine and Specialty Chemicals.

[4] Huang Jianke. 2006. Research progress of meso damage mechanics for ductile fracture criterion in metal forming. Journal of Shanghai Jiaotong University.

[5] Liu Xianlan. 2008. meso damage mechanics and its application in metal plastic forming. Forging Technology.

[6] Gong Yu. 2010. Application of meso damage mechanics in the statistical simulation research of the composite material characteristics. Zhejiang University.

[7] Zhang Yajing. 2009. Research on the architecture design of urban planning exhibition hall. Harbin Institute of Technology.

[8] Guo Donghai. 2011. Research on the architecture design of urban planning exhibition hall in Zhengdong New District. Nanjing University.

[9] Pei Pei. 2013. Research on the architecture lighting and illuminating of the exhibition hall. North Modern Decoration (Theory).

[10] Ma Xinmei. 2014. Research on the architecture planning and exhibition design of the university exhibition hall. Gansu Science and Technology. 